\title{
Errata
}

\section{Information flow due to controlled interference in entangled systems}

\section{R SRIKANTH}

(Pramana - J. Phys., Vol. 59, No. 2, pp. 169-173)

1. In the abstract, the verb 'point out' is to be read 'suggest', the verb 'implies' as 'permits' and 'presented' as 'proposed'.

2. In both equations (4) and (6), the final trignometric factor is to be replaced by $\cos (k[\overline{u x}-\overline{v x}])$.

3. In equation (6), the cosine argument $\left(\phi_{1}-\phi_{4}\right)$ should in fact be half the value, namely $\left(\phi_{1}-\phi_{4}\right) / 2$.

4. The transformation to double slits basis in paragraph 4 , and the right hand side in equation (2) do not represent the complete state, but the part accessible to Bob.

5. In the first sentence beginning after equation (3), please include path segments yrpdhju, yrpdhkv to the list given there.

All conclusions remain intact.

\section{One-dimensional multiple-well oscillators: A time-dependent quantum mechanical approach}

NEETU GUPTA, AMLAN K ROY and B M DEB

(Pramana - J. Phys., Vol. 59, No. 4, pp. 575-583)

On page 578:

1. Table 1, potential parameters $w^{2}=12.0, a_{4}=-2.1, a_{6}=0.18$ :

For $n=0,\left\langle x^{4}\right\rangle=0.265501$, and not 0.265505 .

On page 579:

1. Table 2, potential parameters $w^{2}=20.8, a_{4}=-2.1, a_{6}=0.1$ :

For $n=3,\left\langle x^{8}\right\rangle=2.913128$, and not 0.476101 .

2. Table 2, potential parameters $w^{2}=27.0, a_{4}=-1.5, a_{6}=0.035$ :

For $n=0,\left\langle x^{10}\right\rangle=6434789.3$, and not 643478903 . 